\title{
Tough Transitions: Family Caregiver Experiences with a Pediatric Long Term Ventilation Discharge Pathway
}

\author{
Jackie Chiang$^{1}$, Allia Karim ${ }^{1}$, Andrea Hoffman ${ }^{2}$, Karen Dryden Palmer ${ }^{3}$, Krista Keilty ${ }^{4}$, \\ Faiza Syed ${ }^{5}$, Joanna Janevski ${ }^{1}$, Tilak Dutta ${ }^{6}$, Maryanne Fellin ${ }^{2}$, Sally Lindsay ${ }^{2}$, Laura \\ Beaune $^{2}$, and Reshma Amin ${ }^{5}$ \\ ${ }^{1}$ The Hospital for Sick Children Department of Paediatrics \\ ${ }^{2}$ Holland Bloorview Kids Rehabilitation Hospital \\ ${ }^{3}$ Hospital for Sick Children Research Institute \\ ${ }^{4}$ Hospital for Sick Children \\ ${ }^{5}$ The Hospital for Sick Children \\ ${ }^{6}$ Toronto Rehab
}

May 1, 2021

\begin{abstract}
Objectives: Discharging a child home on long term ventilation (LTV) via tracheostomy is complex and involves multiple healthcare providers across healthcare sectors. To date, patient and family feedback of a newly developed LTV discharge pathway has been anecdotal. Our objective was to explore the perceptions of family caregivers (FCs) that have completed the LTV pathway to home with respect to their: (1) experience with transitions across the pathway (2) perceptions of competency attainment and, (3) viewed opportunities for improvement. Methods: We conducted 11 semi structured interviews with FCs. Interviews focused on FCs experience with the training process, perception of competency from a knowledge and skill perspective and opportunities for improvement. Interviews were audiotaped, transcribed verbatim, coded and analyzed using an inductive thematic analysis approach. Results: Eight mothers and 3 fathers of 10 children participated. Six primary themes were identified: 1) making an informed decision, 2) transitioning to rehabilitation, 3) building capacity for self-care, 4) coordinating case management, 5) readying for discharge home and, 6) experiencing home care. Conclusion: Overall, FCs felt that the preparation and transition support obtained through the application of a standardized LTV discharge pathway allowed successful attainment of new knowledge and skills necessary to care for their child with LTV at home.
\end{abstract}

\section{INTRODUCTION}

Long term ventilation (LTV) at home via tracheostomy represents one of the most advanced, highly specialized and complex therapies offered to individuals outside of a hospital setting. ${ }^{1,2}$ These children are medically fragile with significant associated morbidity and mortality. ${ }^{3,4}$

Notably, the caregiver burden for these families is high. Family caregivers (FC) of children using LTV at home are tasked with providing 'intensive care' in their homes. As such, robust FC competency-based knowledge and skills training must take place in hospital prior to the child's initial discharge home. The published reported median length of stay in hospitals for families to accomplish this preparation is broad ranging from 46 days to 9.6 months. ${ }^{3,5,6-10}$ A handful of studies to date have reported barriers to achieving discharge for this population. , $^{3,5,7,8,11-13}$ These have included delays in organizational decision-making and lack of coordinated approach to care associated with preparation to transition to home.

In partnership with key clinical stakeholders, a LTV discharge pathway was developed in late 2016 to 
restructure and standardize discharge preparation placing emphasis on ensuring quality FC learning and minimizing system borne delay. The pathway was developed to promote safe and timely transitions across three publicly funded but distinct health care organizations that voluntarily collaborate to coordinate care for children on LTV. The pathway starts with a child in an intensive care unit at a tertiary care pediatric hospital followed by transfer to a pediatric rehabilitation hospital and then transition to home. Using a process mapping approach, guided by content experts, the LTV discharge pathway was developed to outline the steps to transition a child newly initiated on LTV from the acute care setting to home. The pathway includes role-based tasks and graduated milestones (see Appendix 1 and 2).

To date, patient and family input into the LTV discharge pathway has been largely anecdotal and never systematically analysed. With the goal of informing a robust family centered LTV pathway, we deigned this study to explore the perceptions of FCs that have completed the LTV discharge pathway with respect to their: (1) experience with transitions across the pathway (2) perceptions of competency attainment (knowledge and skills acquisition) and, (3) opportunities for improvement.

\section{METHODS}

\section{Study Design}

We conducted a qualitative thematic-analysis study comprised of semi-structured interviews with FCs of children requiring LTV support at home. Data was collected between April 2019 and January 2020. Institutional ethics review board approval was received from The Hospital for Sick Children (REB \#1000061042) and Holland Bloorview Kids Rehabilitation Hospital (REB \#18-831). Parents were eligible for study inclusion if they: (1) had a child who was initiated on the LTV discharge pathway between December 2016 and September 2018 and (2) could read and write English. We used purposeful sampling to ensure we captured perspectives of FCs who provide care at home for children with a range of ages and medical conditions that represent varied experiences across the pathway.

\section{Recruitment of Study Participants}

Family caregivers were recruited from the hospital's LTV program clinical database and were introduced to the study via an informational email sent out by a member of the LTV team within the circle of care. A follow up telephone call was conducted by the study's research coordinator where FCs were provided further information about the study, the opportunity to indicate their interest in participating and complete the consenting process if they chose to participate.

\section{Data Collection}

Data collection included individual semi-structured interviews, gathering demographic data and patient medical record review. Interview guides were informed iteratively by the research team after a review of relevant literature. Using concepts of Kirkpatrick's ${ }^{14}$ model of evaluation as a conceptual framework (with a focus on level 1: reactions of learners), a semi-structured interview guide was developed. The 31 question, semi-structured guide explored FC experience in all phases and domains of the LTV discharge pathway and perspectives on where they identified opportunities for improvement (see Appendix 3). Pilot testing of the interview guide was completed with two FCs. The interviews were conducted via telephone by an experienced qualitative researcher. Interviews lasted between 45-60 minutes and occurred at a time that was convenient for the family.

Demographic data was collected during the interview. Data about the child and the medical context of LTV support was retrospectively collected via the hospital's electronic medical database.

\section{Data Analysis}

Interviews were audio recorded, transcribed verbatim and de-identified by a professional transcriptionist. Qualitative data analysis software ${ }^{15}$ was used for data and coding management. An inductive, thematic analysis process to familiarize, code, and identify important themes from the text was used to analyze the data via multiple readings of the transcripts. ${ }^{16}$ The coding authors (JC, RA, LB, AK) engaged in an 
immersive reading of the transcripts. Initial patterns and recurring categories were identified via consensus. Next, similarities and differences between participant accounts in the data were exposed and final thematic codes developed. A codebook was created and iteratively modified. This approach created relationships between concepts or variables that were determined to influence the experiences of FCs with care grounded in the LTV discharge pathway. ${ }^{17,18}$ Methodological rigor was established through prolonged engagement and peer debriefing. ${ }^{19}$ We followed the Consolidated Criteria for Reporting Qualitative studies. ${ }^{20}$ After 11 interviews, thematic saturation had been achieved. ${ }^{17}$

\section{RESULTS}

Thirteen eligible FCs were contacted by the research coordinator. Eleven FCs agreed to be interviewed and two FCs of the same child were interviewed together. See Tables 1 and 2 for demographic characteristics of study participants and their children using LTV. [Table 1, Table 2]

\section{FC Experience Themes}

Six themes emerged from this data that described FC experiences within the LTV discharge pathway (Figure 1). Each theme was associated with a pathway phase from time of choosing to proceed with invasive ventilation up to the point of caring independently for their child at home. These themes were: 1) making an informed decision, 2) transitioning to rehabilitation, 3) building capacity for self-care, 4) coordinating case management, 5) readying for discharge home and, 6) experiencing home care.

Making an informed decision

The way in which health care providers (HCPs) share and discuss a child's diagnosis and decision for LTV is a critical touchpoint in building FC trust in the LTV discharge plan. FCs expressed that this was a time of both emotional and informational overload for them. They identified that characteristics of information they perceived as helpful were hopeful, family centred and consistent. FCs explained that it was difficult to hear acute care HCPs share information about their child's prognosis that they experienced as fatalistic. They expressed a desire for their child's prognosis to be viewed from a holistic perspective. Access and interactions with LTV and clinical disease experts instilled parental confidence and reassured FCs that they made the correct decision to pursue LTV for their child. Factors that inhibited this process were a perceived lack of communication amongst various clinical teams, informational gaps, and misinformation. They also expressed the need to connect with families in similar situations, for enhanced emotional support (see Appendix 4).

Transitioning to rehabilitation

FCs expressed that moving from an acute intensive care setting to a rehabilitative setting raised fear and anxiety because of potentially different organizational structure and teaching approaches. They explained that their concerns were eased by effective team communication as well as transitioning into a more active parenting role which they linked to increasing their confidence and capacity to care for their child. Challenges occurred when teaching practices differed between organizations. FCs recommended family involvement in transition meetings, a key HCP contact in acute care to accompany the family to the rehabilitation setting to support continuity of care both from a process and physical lens (see Appendix 5).

Building capacity for self-care

FCs provided valuable insights into effective strategies that supported their capacity in preparing to care for their child at home. Comprehensive training was instrumental to their perceived readiness to care for their child upon discharge home. The identified key components included: 1) training by consistent content experts (nurses and respiratory therapists), 2) experiential or "hands-on" learning approach, 3) parent led pace, 4) knowledge demonstration and 5) adaptive and flexible sessions. In the acute care setting, there was a lack of opportunity for "hands-on" teaching. However, they also self-identified that immediately after tracheostomy insertion, their emotional state hindered readiness to learn while in acute care (see Appendix $6)$.

Coordinating case management 
FCs described that they were unprepared and overwhelmed by financial costs. These related to the cost of equipment and supplies. Although efforts were made to connect and organize respiratory vendors with FCs prior to discharge, FCs felt that they did not have enough information or expertise with managing this aspect of care. They reported the need for experiential advice from other FCs who could help guide them in determining how to get things set up at home (see Appendix 7).

Readying for Discharge Home

FCs expressed their feelings of competence in caring for their child at home along with its many challenges. Common across all FCs were the experiences of constant worry about the "what if's" such as an emergency event with their child. FCs described feeling that HCPs had encouraged them to maintain a 24/7 vigilance over their child's care which FCs experienced as unsustainable. Loss of hospital expertise and after hours support for guidance and problem solving were described as extremely concerning for FCs. The stress of having to turn their home into a safe and ongoing medical space for their child surfaced throughout the interviews (see Appendix 8).

Experiencing home care

FCs shared their experiences with respect to helpful and unhelpful in-home support. Many FCs cited challenging experiences with nursing support at home including unavailability of nurses and their lack of experience with children, tracheostomy tubes or emergency situations. FCs found tremendous benefit in connecting with other families through on-line support groups where they often learned care tips. Some FCs flagged opportunities for re-education and training post discharge as their child's development and care needs changed (see Appendix 9).

\section{DISCUSSION}

To our knowledge, we are reporting on the first Canadian data exploring the experiences of FCs of children requiring LTV via tracheostomy transitioned from hospital to home within a newly developed pediatric LTV discharge pathway framework. An emerging model of factors that are reported to impact FC experience with the LTV pathway was developed as an outcome of our study (Figure 2).

Our study provides new insight into the experiences of FCs with their HCPs and information sharing. Several FCs in our study perceived that information sharing by HCPs about their child's life with LTV was "doom and gloom". These findings are similar to a recent study of children with medical complexity in which FCs stated that they preferred that broad Advance Care Planning conversations to be holistic and hopeful. ${ }^{21}$ Additionally, in a study that explored the perspectives of North American directors of pediatric home ventilation programs, the authors found that negative bias about the quality of life and abilities of children with severe disabilities posed a barrier to FC decision making in pursuing LTV and recommended HCP education to reduce this inherent bias. ${ }^{22}$ FCs in our study reported that they preferred to have conversations with HCPs who were more familiar with the experience of children with LTV at home and who were aware of the range of possibilities for their child's quality of life, happiness and life at home.

Shared decision making in paediatric health is grounded in a family centred framework. It centres on the FC and HCP partnership and respect for FC goals and guides a collaborative effort to come to an agreement on the child's plan of care. ${ }^{23-25}$ Barriers to shared decision making from the FC perspective occur when information is not shared early enough in the child's illness trajectory, conversations are not conducted with HCPs who have sufficient knowledge of the family, HCPs provide inconsistent information and/or lack capacity to address cultural differences. ${ }^{21,22,26}$ Many FCs in our study experienced barriers to shared decision making such as inadequate information about the meaning of LTV for their child and lack of access to LTV or disease-specific experts to address their questions. FCs in this study recommended having access to peer support from families with children who had similar care needs.

There is a small but growing body of research exploring the experiences of transitions from hospital to home of FCs supporting individuals with LTV needs. ${ }^{27-30}$ A literature review of 14 studies found that overall FCs demonstrated competence in caring for their child with LTV needs at home but that resounding themes 
included feelings of isolation when transitioning to home from hospital. ${ }^{28} \mathrm{FCs}$ in our study reported that through the LTV discharge pathway, they had acquired competency and skills regarding the routine medical needs required by their child at home. FC reports of knowledge and capacity attainment have been previously linked to teaching by ventilator experts, opportunities to demonstrate self-efficacy prior to discharge home ${ }^{1}$ and a learning process cycle ${ }^{30}$ all of which were substantiated in this study.

The initial discharge home was noted to be particularly stressful for FCs. Expectations with respect to being fully responsible for the child's medical needs at home along with providing continuous $24 / 7$ care created stress and fear. The negative impact on the health and well-being of FCs caring for technologically dependent children at home has been well documented to include higher risks of sleep deprivation, depression and lowered health related quality of life and family functioning. ${ }^{31}$ Although the experiences of FCs in our study over time were not examined, emotional distress and concerns regarding sleep quality were identified.

FC anxiety were exacerbated by being disconnected from familiar hospital HCPs post discharge. Direct access to hospital experts within the first several weeks after transitioning home was reported as positively impacting problem solving, consolidating learned knowledge and enhanced self-efficacy for ventilator assisted adults transitioning to home ${ }^{1}$ We recommend a formal extension of the LTV discharge pathway activities into the first month that includes a telephone follow-up prior to the first LTV clinic visit. In addition, connecting FCs to families of children with LTV who have successfully transitioned home early in the pathway process may be beneficial in terms of helping families understand and prepare for the challenges of being home and navigating coordination of care. ${ }^{30}$

One of the biggest challenges reported by FCs in our study and previously documented by others $3,29,30,32$ are issues related to gaps in services in the home setting. In our study, FCs discussed the additional burden of care related to supervising home care nurses as well as the lack of consistent overnight nursing. ${ }^{33}$ FCs also discussed the incredible amount of time spent on case management including dealing with equipment and supplies. We recommend innovative partnerships with home care nursing agencies to support the training of community nurses which may include matching community nurses to FCs who are learning LTV care in the hospital to support dual learning and relationship building that is continued into the child's home. Mobile training such as joint medical simulation may be useful for training in the home for home care nurses and continued learning for FCs. ${ }^{30}$ Given the uptake of new electronic health technologies, opportunities to virtually access the inside of a family's home, may help HCPs and peer families guide FCs in setting up medical equipment, organizing their home and addressing safety procedures, thus providing an additional layer of support for families. ${ }^{30}$ [Table 3]

Our study did have some notable limitations. The research was conducted at one point in time and subject to participant recall bias. All FC participants were English-speaking, predominantly mothers, and from welleducated, middle- to high-income families. Although in the sampling strategy, participants were purposefully selected to reflect the diversity of families who live in Toronto, Ontario, Canada, our study results may not reflect the experiences of other socio-economically or linguistically diverse families. It may also not be generalizable to other LTV programs given the different infrastructure and operations that exist nationally and internationally. As such, the caregiver training procedures, community resources and supports for caregivers will vary although the guiding principles would all be the same.

\section{CONCLUSION}

FCs of children using LTV willingly take on the intensive medical care that their child requires to live at home. To quote one of the FC's interviewed in the study "it's a massive undertaking when you sit back and reflect on what you do... You're actually a full-blown [health care] professional yourself, but it is full time care".

FCs reported that they need to be prepared in all aspects of 'what life will be like' living with a child with LTV care needs at home. They reported a positive impact of knowledge and skills learned but also recommended that decisions to implement the LTV pathway should be framed as hopeful and holistic. FCs favored a teaching approach that was comprehensive, flexible and family centred. FCs in this study highlighted the 
need for enhanced support with respect to the child's care coordination and access to skilled and consistent in-home nursing support. They also shared the need for continued support of hospital experts beyond the child's discharge from hospital to ensure continuity of care, family well-being, and continued opportunities to build on self-efficacy.

\section{ACKNOWLEDGEMENTS:}

The authors thank all participants including patients, parents, health care providers, administrators and community partners who participated in or contributed to the study.

\section{REFERENCES}

1. Dale CM, King J, Nonoyama M, Carbone S, McKim D, Road J, Rose L. Transitions to Home Mechanical Ventilation. The Experiences of Canadian Ventilator-assisted Adults and Their Family Caregivers. Ann Am Thorac Soc. 2018 Mar;15(3):357-64.

2. Wallis C, Paton JY, Beaton S, Jardine E. Children on long-term ventilatory support: 10 years of progress. Arch Dis Child. 2011;96:998-1002.

3. Edwards EA, Hsiao K, Nixon GM. Paediatric home ventilatory support: the Auckland experience. J Paediatr Child Health. 2005;41:652-658.

4. Edwards JD, Kun SS, Keens TG. Outcomes and causes of death in children on home mechanical ventilation via tracheostomy: an institutional and literature review. J Pediatr. 2010;157:955-959.

5. Oktem S, Ersu R, Uyan ZS, et al. Home ventilation for children with chronic respiratory failure in Istanbul. Respiration; international review of thoracic diseases. 2008;76:76-81.

6. Appierto L, Cori M, Bianchi R, et al. Home care for chronic respiratory failure in children: 15 years experience. Paediatr Anaesth. 2002; 124: 345-350.

7. Graf JM, Montagnino BA, Hueckel R, McPherson ML. Children with new tracheostomies: Planning for family education and common impediments to discharge. Pediatr Pulmonol. 2008; 438: 788-794.

8. DeWitt PK, Jansen MT, Ward SL, Keens TG. Obstacles to discharge of ventilator-assisted children from the hospital to home. Chest. 1993; 1035: 1560-1565.

9. Tearl DK, Cox TJ, Hertzog JH. Hospital discharge of respiratory technology-dependent children: Role of a dedicated respiratory care discharge coordinator. Respir Care. 2006; 517: 744-749.

10. Fraser J, Henrichsen T, Mok Q, Tasker RC. Prolonged mechanical ventilation as a consequence of acute illness. Arch Dis Child. 1998; 783: 253-256.

11. Amin R, Sayal A, Syed F, et al. How Long Does It Take to Initiate a Child on Long-term Invasive Ventilation: Results from a Canadian Paediatric Home Ventilation Program. Can Respir J. 2015; 22: 103108.

12. Goldberg AI, Frownfelter D. The Ventilator-assisted Individuals Study. Chest. 1990; 982: 428-433.

13. Noyes J. Barriers that delay children and young people who are dependent on mechanical ventilators from being discharged from hospital. J Clin Nurs. 2002; 111: 2-11.

14. Kirkpatrick DL. Evaluation of training. In R. L. Craig (Ed.), Training and development handbook: A guide to human resource development. New York (NY): McGraw Hill; 1976.

15. NVivo qualitative data analysis software. Version 9 [Computer Software]. Doncaster (AU): QSR International Pty Ltd; 2007.

16. Braun V \& Clarke V. Using thematic analysis in psychology. Qual Res in Psychol. 2006;3(2):77-101. 
17. Padgett DK. Strategies for Rigor. In: Qualitative Methods in Social Work Research, 2nd ed. Thousand Oaks (CA): Sage Publications Inc; 2008. p 179-198.

18. Tuckett A. Rigour in qualitative research: Complexities and solutions. Researcher 2005;13:29-42.

19. Patton M. Purposeful Sampling. In: Qulitative evaluation and research methods. Beverly Hills (CA): Sage; 1990. p 169-186.

20. Tong A, Sainsbury P, Craig J. Consolidated criteria for reporting qualitative research (COREQ): a 32-item checklist for interviews and focus groups. Int J Qual Health Care. 2007;19(6):349-357.

21. Orkin J, Beaune L, Moore C, Weiser N, Arje D, Rapoport A, Netten K, Adams S, Cohen E, Amin R. Toward an Understanding of Advance Care Planning in Children with Medical Complexity. Pediatrics. 2020 Mar 1;145(3).

22. Edwards JD, Morris MC, Nelson JE, Panitch HB, Miller RL. Decisions around long-term ventilation for children. Perspectives of directors of pediatric home ventilation programs. Ann Am Thorac Soc. 2017 Oct;14(10):1539-1547.

23. Adams RC, Levy SE. Shared decision-making and children with disabilities: pathways to consensus. Pediatrics. 2017 Jun 1;139(6): e20170956.

24. Dunst CJ, Trivette CM, Hamby DW. Metaanalysis of family-centered helpgiving practices research. Ment Retard Dev Disabil Res Rev. 2007;13(4):370-378.

25. Kuo DZ, Houtrow AJ, Arango P, Kuhlthau KA, Simmons JM, Neff JM. Familycentered care: current applications and future directions in pediatric health care. Matern Child Health J. 2012;16(2):297-305.

26. Edwards JD, Panitch HB, Nelson JE, Miller RL, Morris MC. Decisions for long-term ventilation for children: perspectives of family members. Ann Am Thorac Soc. 2020 Jan;17(1):72-80.

27. Edwards EA, O'Toole M, Wallis C. Sending children home on tracheostomy dependent ventilation: pitfalls and outcomes. Arch Dis Child. 2004;89:251-255.

28. Condon A. Parents' perceptions of the transition of their child who is ventilator dependent from hospital to home. Nurs Child Young People. 2020 Jan 9;32(1).

29. Park M, Jang H, Sol IS, Kim SY, Kim YS, Kim YH, Sohn MH, Kim KW. Pediatric home mechanical ventilation in Korea: the present situation and future strategy. J Korean Med Sci. 2019 Oct 1;34(42).

30. Amar-Dolan LG, Horn MH, O'Connell B, Parsons SK, Roussin CJ, Weinstock PH, Graham RJ. "This Is How Hard It Is": Family Experience of Hospital-to-Home Transition with a Tracheostomy. Ann Am Thorac Soc. $2020 \mathrm{Jul} ; 17(7): 860-868$.

31. Chan YH, Lim CZ, Bautista D, Malhotra R, Østbye T. The health and well-being of caregivers of technologically dependent children. Glob Pediatr Health. 2019 Jan; 6: 2333794X18823000.

32. Whiting, M. Caring for children - '24-7': The experience of WellChild Nurses and the families for whom they are providing care and support. J Child Health Care. 2018 May 17;23(1):35-44.

33. Amin R, Sayal P, Syed F, Chaves A, Moraes TJ, MacLusky I. Pediatric long-term home mechanical ventilation: Twenty years of follow-up from one Canadian center. Pediatr Pulmonol. 2014 Aug;49(8):816-24.

\section{TABLES}

Table 1. FC Demographic Characteristics

Insert Table 1

Table 2. Child with LTV Demographic Characteristics 
Insert Table 2

Table 3. Recommendations for Hospital to Home LTV discharge pathway

Insert Table 3

\section{FIGURES}

Figure 1. Overall Thematic Framework

Insert Figure 1

Figure 2. Facilitators of Hospital to Home LTV Discharge Pathway

Insert Figure 2

\section{Hosted file}

LTV Tables_April 22 2021.pdf available at https://authorea.com/users/411318/articles/520394tough-transitions-family-caregiver-experiences-with-a-pediatric-long-term-ventilationdischarge-pathway

Figure 1. Overall Thematic Framework
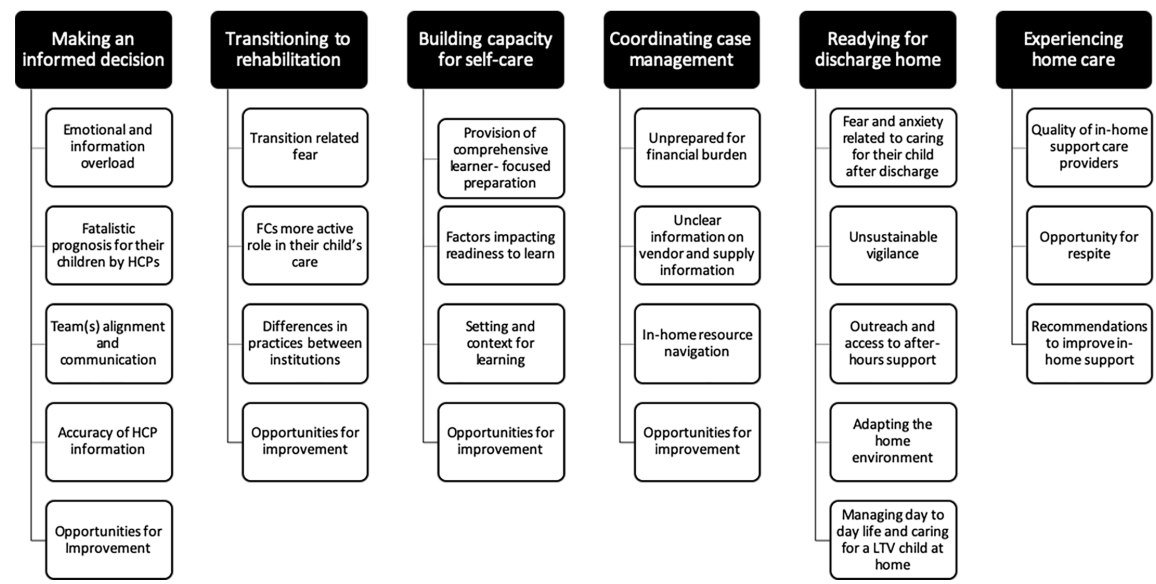

Figure 2. Facilitators of Hospital to Home LTV Discharge Pathway

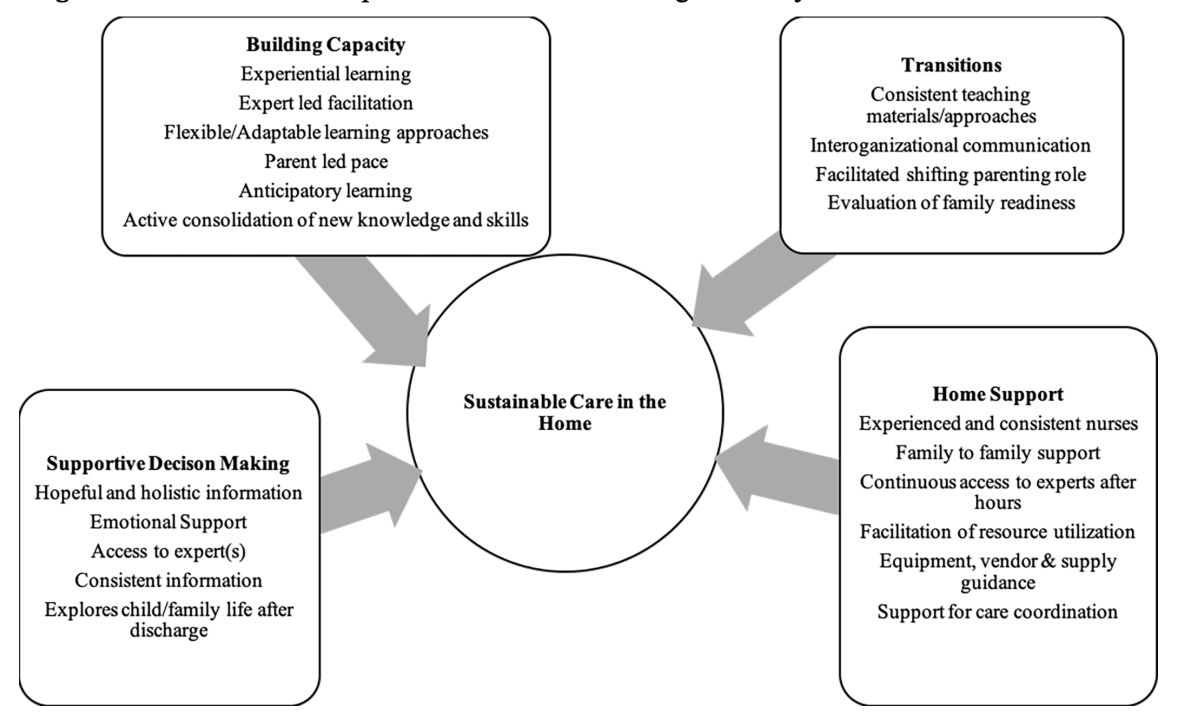

